\title{
Diagnostic and prognostic potential of serum miR-7, miR-16, miR-25, miR-93, miR-182, miR-376a and miR-429 in ovarian cancer patients
}

\author{
Xiaodan Meng ${ }^{1}$, Simon A Joosse ${ }^{1}$, Volkmar Müller ${ }^{2}$, Fabian Trillsch², Karin Milde-Langosch ${ }^{2}$, Sven Mahner ${ }^{2}$, \\ Maria Geffken ${ }^{3}$, Klaus Pantel ${ }^{1}$ and Heidi Schwarzenbach ${ }^{* 1}$ \\ ${ }^{1}$ Department of Tumor Biology, University Medical Center Hamburg-Eppendorf, Hamburg 20246, Germany; ${ }^{2}$ Department of \\ Gynecology, University Medical Center Hamburg-Eppendorf, Hamburg 20246, Germany and ${ }^{3}$ Department of Transfusion \\ Medicine, University Medical Center Hamburg-Eppendorf, Hamburg 20246, Germany
}

Background: Owing to late diagnosis in advanced disease stages, prognosis of patients with epithelial ovarian cancer (EOC) is poor. The quantification of deregulated levels of microRNAs could facilitate earlier diagnosis and improve prognosis of EOC.

Methods: Seven microRNAs (miR-7, miR-16, miR-25, miR-93, miR-182, miR-376a and miR-429) were quantified in the serum of 180 EOC patients and 66 healthy women by TaqMan PCR microRNA assays. Median follow-up time was 21 months. The effects of miR-7 and miR-429 on apoptosis, cell proliferation, migration and invasion were investigated in two (EOC) cell lines.

Results: Serum levels of miR-25 $(P=0.0001)$ and miR-93 $(P=0.0001)$ were downregulated, whereas those of miR-7 $(P=0.001)$ and miR-429 ( $P=0.0001)$ were upregulated in EOC patients compared with healthy women. The four microRNAs discriminated EOC patients from healthy women with a sensitivity of $93 \%$ and a specificity of $92 \%$. The levels of miR-429 positively correlated with CA125 values $(P=0.0001)$ and differed between FIGO I-II and III-IV stages $(P=0.001)$. MiR-429 was an independent predictor of overall survival $(P=0.011)$. Overexpressed miR-429 in SKOV3 cells led to suppression of cell migration $(P=0.037)$ and invasion $(P=0.011)$. Increased levels of miR-7 were associated with lymph node metastases $(P=0.0001)$ and FIGO stages III-IV $(P=0.0001)$. Overexpressed miR-7 in SKOV3 cells resulted in increased cell migration $(P=0.001)$ and invasion $(P=0.011)$. Additionally, the increased levels of miR-376a correlated with FIGO stages III-IV $(P=0.02)$.

Conclusions: Our data indicate the diagnostic potential of miR-7, miR-25, miR-93 and miR-429 in EOC and the prognostic potential of miR-429. This microRNA panel may be promising molecules to be targeted in the treatment of EOC.

Epithelial ovarian cancer (EOC) is the most lethal gynecological cancer in the western world. The high mortality may also be due to the fact that EOC is usually detected too late. Approximately $70 \%$ of patients are diagnosed with advanced FIGO stages (III or IV) and have a 5 -year survival rate of $<40 \%$, whereas patients who are diagnosed with FIGO stage I or II have a longer 5-year survival rate of $70-90 \%$. Current diagnostic methods for detection and monitoring of EOC mainly include pelvic examination and transvaginal ultrasound and measurement of serum biomarker CA125 (carbohydrate antigen 125) (Jayson et al, 2014). However, these methods are not sufficiently specific to diagnose EOC at an early stage, as, for example, CA125 is only elevated in approximately $50 \%$ of stage I and in $70-90 \%$ of advanced diseases (Meinhold-Heerlein and Hauptmann, 2014). Thus sensitive, noninvasive biomarkers might help to improve early detection and to guide therapy.

Such markers could be microRNAs (miRNAs). These small regulatory and non-coding RNA molecules consist of approximately 22 nucleotides (Davidson et al, 2014). They inhibit the gene expression posttranscriptionally by binding specifically to the

*Correspondence: Assistant Professor Dr H Schwarzenbach; E-mail: hschwarz@uke.uni-hamburg.de

Received 6 May 2015; revised 24 August 2015; accepted 28 August 2015; published online 22 September 2015

(C) 2015 Cancer Research UK. All rights reserved 0007 - 0920/15 
$3^{\prime}$ untranslated-region ( $3^{\prime} \mathrm{UTR}$ ) of their target mRNAs. Gene silencing can occur through the translational inhibition or cleavage of their target mRNAs depending on the complementary sequence between binding sites of the specific mRNAs and miRNAs (Bartel, 2009). One miRNA can potentially bind to hundreds of target genes and is, therefore, involved in the regulation of various cellular processes, such as development, differentiation and proliferation (Cheng et al, 2005). As miRNA loci frequently map to fragile chromosomal regions harbouring DNA amplifications, deletions or translocations, their expression is often deregulated during tumorigenesis (Heneghan et al, 2010). MiRNAs are released into the blood circulation by active cell secretion or by apoptotic and necrotic cells (Schwarzenbach et al, 2011) and exist there either cell freely, associated with Argonaut or HDL proteins, or in exosomes (Simpson et al, 2009).

We, therefore, selected miR-7, miR-16, miR-25, miR-93, miR182, miR-376a and miR-429 for our study, because these miRNAs have cancer-specific characteristics that could also be interesting for EOC. The aim of this study was to investigate the expression pattern of this miRNA panel in a large EOC patient cohort with a long follow-up and whether its signature may serve as a minimally invasive diagnostic and prognostic marker for EOC. We also compared these serum miRNAs with serum CA125, in particular to increase the sensitivity of EOC detection in EOC patients.

\section{MATERIALS AND METHODS}

Study populations. Serum samples from 180 consecutive EOC patients treated at the University Medical Center HamburgEppendorf, Department of Gynecology for histologically confirmed International Federation of Gynecology and Obstetrics (FIGO) stages I-IV were included. Mean and median ages were 60 and 62 years, respectively, and ranged between 25 and 91 years. Borderline ovarian tumours or benign ovarian tumours were not part of this study. Blood serum samples were collected directly before surgery of the primary tumour between February 1997 and April 2013. All patients gave written informed consent to access their blood samples and review their medical records according to our investigational review board and ethics committee guidelines. Regarding blood processing, uniform management concerning the specific described protocols was performed. The median follow-up time was 21 months (range 1-176 months). Patients were treated according to national guidelines (Wagner et al, 2013). First-line treatment after primary surgery consisted of carboplatin and paclitaxel. Detailed patient characteristics are summarised in Table 1.

In addition, serum samples were collected from 66 age-matched (mean age of 59 years and median age of 57 years, range 37-85 years) healthy women with no history of any cancer and in good health based on self-report. This group served as a control population. Blood collection and experiments were performed in compliance with the Helsinki Declaration and were approved by the ethics committee (Ethik-Kommission der Ärztekammer Hamburg, Hamburg).

Extraction of small RNA and conversion into cDNA. Small RNA was extracted from $300 \mu \mathrm{l}$ serum using the NucleoSpin miRNA Plasma Kit (Macherey-Nagel, Düren, Germany) and according to the manufacturer's instructions. For extraction efficiency, $20 \mathrm{fmol}$ of synthetic non-human cel-miR-39 was added as an exogenous spike in control. Reverse transcription was performed by the TaqMan miRNA Reverse Transcription Kit (Life Technologies, Grand Island, NY, USA) and according to the manufacturer's instructions.

Preamplification of miR-7, miR-182, miR-376a and miR429. Owing to the low expression levels of miR-7, miR-182,
Table 1. Characteristics at the time of first diagnosis of EOC patients

\begin{tabular}{|l|c}
\hline Parameters & Patients (\%) \\
\hline EOC patients & $180(100)$ \\
\hline Age & 60 years (range 25-91 years) \\
\hline
\end{tabular}

\section{FIGO stage}

FIGO I-II

FIGO III-IV

Unknown

$32(17.8)$

$147(81.7)$

$1(0.5)$

\section{Grading}

Low grade

High grade

Unknown

57 (31.7)

$120(66.7)$

$3(1.6)$

Lymph node metastasis

\begin{tabular}{|l|r}
\hline N0 & $51(28.3)$ \\
N1 & $88(48.9)$ \\
\hline
\end{tabular}

Unknown

41 (22.8)

CA125, $\mathrm{Uml}^{-1}$

\begin{tabular}{|c|r}
\hline$<35$ & $13(7.2)$
\end{tabular}

$35-65$

$>65$

Unknown

7 (3.9)

$148(82.2)$

Overall survival

\section{Dead}

Alive

Unknown

$12(6.7)$

\section{Recurrence}

\begin{tabular}{|l|c}
\hline Yes & $97(53.9)$ \\
No & $82(45.6)$ \\
Unknown & $1(0.5)$
\end{tabular}

\section{Tumour residual}

\begin{tabular}{|l|r}
\hline Tumor-free & $125(69.4)$
\end{tabular}

$<1 \mathrm{~cm}$

$>1 \mathrm{~cm}$

Unknown

27 (15.0)

$19(10.6)$

Healthy women

$9(5)$

Age

$66(100)$

Abbreviations: $\mathrm{CA} 125=$ carbohydrate antigen $125 ; \mathrm{EOC}=$ epithelial ovarian cancer; FIGO = International Federation of Gynecology and Obstetrics.

miR-376a and miR-429 in EOC patients and healthy women, a preamplification of its cDNA was performed. For an accurate normalisation of these miRNAs, cDNA of the reference miR-484 was also preamplified. To avoid false positive data (e.g., primer dimer formation or unspecific PCR products) derived from preamplification or real-time PCR, a negative control without any templates was included from the starting point of reverse transcription. To check whether the PCR products are real amplification products, we analysed them by agarose gel electrophoresis.

Quantitative real-time PCR of miR-7, miR-16, miR-25, miR-93, miR-182, miR-376a and miR-429. The miRNA-specific TaqMan miRNA assays (Life Technologies) for miR-484 (reference miRNA), miR-7, miR-16, miR-25, miR-93, miR-182, miR-376a and miR-429 were used, and each miRNA was quantified by realtime PCR in triplicate assay, as described in our previous article (Joosse et al, 2014).

Normalisation and data analyses. There is no consensus concerning the normalisation of circulating miRNAs. Therefore, we described the shortcomings on the data normalisation by a reference miRNA in our review article (Schwarzenbach et al, accepted by Clin Chem). The wrong choice of a reference miRNA can have serious impact on the actually available miRNA transcript levels and consequently on the biological interpretation of data. We, therefore, chose miR-484 as reference to normalise our 
miRNA data, because this miRNA showed the smallest variations. In the serum samples of EOC patients and healthy women, we calculated a mean value of $16.92 \quad($ s.d. $=1.41$ ) and 16.18 $($ s.d. $=1.08)$, respectively. Based on its stable expression, miR-484 has also been recommended as a reference gene in the serum of breast cancer ( $\mathrm{Hu}$ et al, 2012). The obtained data of the miRNA expression levels were calculated and evaluated by the $\Delta \mathrm{Cq}$ method as follows: $\Delta \mathrm{Cq}=$ mean value $\mathrm{Cq}$ (reference miR-484) - mean value $\mathrm{Cq}$ (miRNA of interest) and the relative miR levels corresponded to the value of $2^{(\Delta \mathrm{Cq})}$. The interindividual variability of the efficiency of our procedures was also controlled by spiking of cel-miR 39. Our measurements showed a mean value of 21.69 with an s.d. of 0.82 and a median value of 21.66 , indicating that our data are relatively robust.

Cell lines and transient transfection. The cell lines SKOV 3 and OVCAR8 were cultured in McCoy's 5A modified medium and RPMI 1640 with GlutaMAX medium (Life Technologies), respectively, supplemented with $10 \%$ FCS (fetal calf serum; PAA Laboratories, Cölbe, Germany) under standard conditions $\left(37^{\circ} \mathrm{C}\right.$, $5 \% \mathrm{CO}_{2}$, humidified atmosphere). SKOV3 and OVCAR8 cells were transfected with the double-stranded miScript miRNA mimic miR7 or miR-429 at final concentrations of $10 \mathrm{~nm}$ (Qiagen, Hilden, Germany) or with the single-stranded miScript inhibitor miR-7 or miR-429 or scrambled (negative) miRNA control at final concentration of $50 \mathrm{~nm}$ (Qiagen) with $2 \mu \mathrm{l}$ X-tremeGENE HP DNA Transfection Reagent (Roche Diagnostics, Mannheim, Germany).

Apoptosis assay, flow cytometry and MTT assays. These assays are described in the Supplementary Materials.

Cell migration and invasion assays. Cell migration and invasion were performed using $8-\mu \mathrm{m}$ pore uncoated or BME (basement membrane extract)-coated transwell inserts, respectively (Trevigen, City of Gaithersburg, MD, USA), as described in our previous work (Eichelser et al, 2014).

Statistical analyses. The statistical analyses were performed using the SPSS software package, version 18.0 (SPSS Inc., Chicago, IL, USA) and MatLab R2012b (The MathWorks Inc., Natick, MA, USA). Relative expression data were log2 transformed in order to obtain normally distributed data. Statistical difference of miRNA expressions between healthy controls and EOC patients were calculated using ANOVA with Tukey's HSD test for all pairwise comparisons that correct for experiment-wise error rate. Twosample comparisons were performed using Student's $t$-test for equal or unequal variance where appropriate, and the Holm-Bonferroni method was employed for multiple comparison correction. Predictive value of expression data was performed using leaveone-out cross-validation in multinomial logistic regression. Bivariate analyses of the Spearman-Rho test were used. Diagnostic power of the miRNAs was analysed by receiver operating characteristic (ROC) curves. Areas under the curves (AUC) were calculated, assuming nonparametric distribution. Univariate and multivariate analyses were performed for prognostic factors of overall survival using the Cox regression model. Kaplan-Meier plots were drawn on to estimate overall and disease-free survival, and the log-rank test was applied for statistical analyses. Missing data were handled by pairwise deletion. A $P$-value $<0.05$ was considered statistically significant. All $P$-values are two sided.

\section{RESULTS}

Quantification of serum miR-7, miR-16, miR-25, miR-93, miR182, miR-376a and miR-429. The reason for this miRNA selection was that miR-7 is able to inhibit tumour metastasis and reverse epithelial-mesenchymal transition (EMT) through AKT and ERK1/2 pathway inactivation by reducing EGFR expression in
EOC cell lines (Zhou et al, 2014). There are discrepant findings on miR-16. This miRNA has been described to be a reference gene for data normalisation (McDermott et al, 2013) or be deregulated in different cancer types (Cui, 2015; Stuckrath et al, 2015). MiR-25 and miR-93 are involved in proliferation, migration and invasion and have diagnostic and prognostic potential in various cancer types (Smith et al, 2012; Komatsu et al, 2014; Li et al, 2015). MiR182 is involved in aggressive EOC (Xu et al, 2014). MiR-376a can regulate proliferation, apoptosis, migration and invasion in metastatic prostate cancer cells (Formosa et al, 2014). Finally, miR-429 is involved in mesenchymal-epithelial transition (MET) by targeting ZEB1 and ZEB2 (Gregory et al, 2008). To date, circulating, cell-free transcript levels of our miRNA panel have only been quantified for miR-93 in the serum of a small cohort of EOC patients (Ji et al, 2014). The particular features of these miRNAs provoked us to analyse their profile in EOC serum.

The transcript levels of total small RNA and our miRNA panel (miR-7, miR-16, miR-25, miR-93, miR-182, miR-376a and miR429) were quantified in the serum samples of 180 EOC patients and 66 age-matched healthy women by NanoDrop Spectrophotometer and TaqMan PCR, respectively. The total small serum RNA levels were significantly higher in EOC patients than in healthy women $(P=0.0001$, Supplementary Figure $S 1)$. The serum levels of circulating miR-7 $(P=0.001)$ and miR-429 $(P=0.0001)$ were significantly higher, whereas the levels of miR-25 $(P=0.0001)$ and miR-93 $(P=0.0001)$ were significantly lower in EOC patients compared with healthy women. Moreover, the levels of miR-376a were only slightly increased $(P=0.049)$, while the levels of miR-16 and miR-182 were similar between the patient cohort and healthy controls (Figure 1A). AUC values of upregulated miR-7 and miR429 were 0.675 and 0.845 (Figure 1B), respectively, while those of downregulated miR-25 and miR-93 were 0.834 and 0.790 (Figure 1C), respectively, showing the significant difference of the transcript concentrations between EOC patients and healthy women.

For discriminant analysis between healthy women and EOC patients, we used the panel of the four significant miRNAs (miR-7, miR-25, miR-93 and miR-429) and randomly selected $60 \%(n=108)$ of the patient sample cohort as a training set for the binomial logistic regression-based classifier. The remaining $40 \%(n=72)$ was used as an independent test set, resulting in a sensitivity of $93 \%$ and a specificity of $92 \%$ to detect EOC by this miRNA panel (Figure 1D). Sensitivities and specificities of each miRNA and the miRNA panel were determined by the highest Youden index (sensitivity + specificity -1 ) (Figure 1E).

Correlation of the miRNAs with clinicopathological features. These findings provoked us to investigate whether the changes in the concentrations of these miRNAs correlate with the values of tumour marker CA125. Serum CA125 assessment is currently the standard of care in performing diagnosis, following response to treatment, and predicting prognosis of EOC patients. Its sensitivity increases during cancer progression (Meinhold-Heerlein and Hauptmann, 2014). We detected that only miR-429 concentrations correlated with CA125 values ( $r=0.379, P=0.0001$, Figure $2 \mathrm{C})$. As no CA125 values were measured in healthy women, we could not determine and compare its sensitivity and specificity with those of serum miR-429 to detect EOC. Moreover, the patient subgroups harbouring CA125 values of $<35 \mathrm{U} \mathrm{ml}^{-1}$ (normal range, $n=13$ ) or CA125 values of $35-65 \mathrm{U} \mathrm{ml}^{-1}$ (threshold range, $n=7$ ) were too small to statistically evaluate the relative data of miR-429 in these subgroups in comparison with the healthy population. However, we detected that patients with normal and threshold CA125 values had about two- and three-fold higher mean serum levels of miR-429 than healthy women $(n=66)$, respectively (data not shown). 


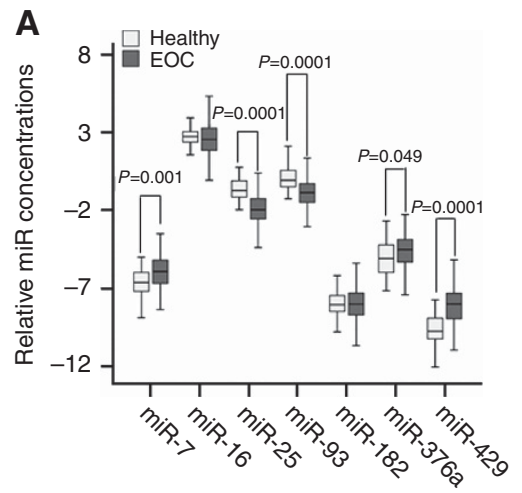

D

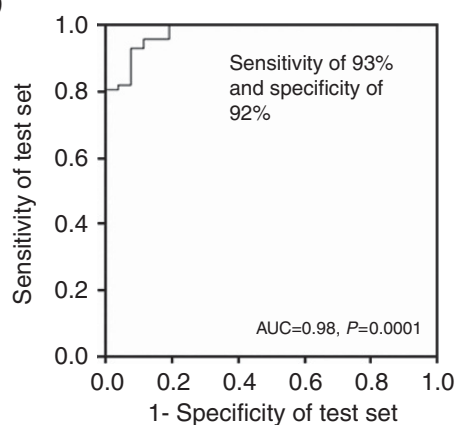

B

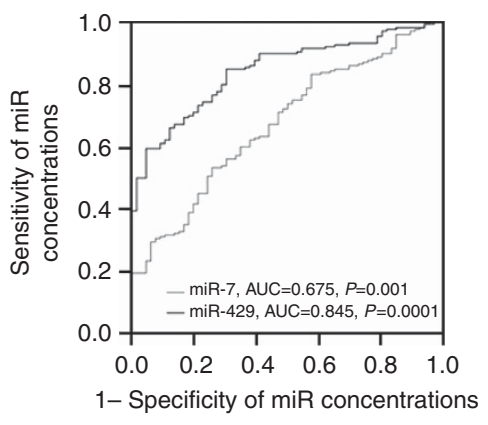

C

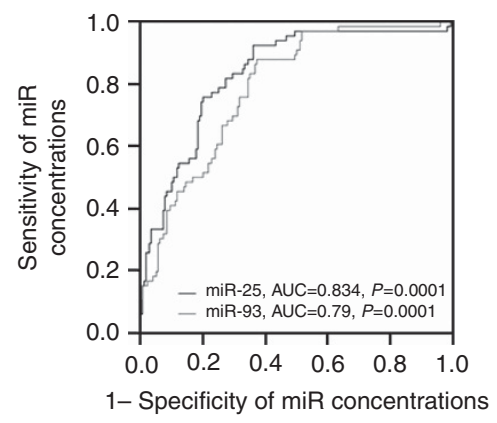

E

\begin{tabular}{lcc} 
EOC $v s$ healthy & Sensitivity & Specificity \\
\hline miR-7 & 0.533 & 0.742 \\
miR-25 & 0.924 & 0.639 \\
miR-93 & 0.879 & 0.628 \\
miR-429 & 0.594 & 0.955 \\
miR-7, -25, -93 and -429 & 0.930 & 0.920 \\
\hline
\end{tabular}

Figure 1. Quantification of miR-7, miR-16, miR-25, miR-93, miR-182, miR-376a and miR-429 in the serum of EOC patients. (A) The box plot compares the miRNA concentrations in the serum of healthy women $(n=66)$ with EOC patients $(n=180)$. ROC analyses show the profiles of sensitivity and specificity (B) of miR-7 and miR-429 and (C) of miR-25 and miR-93 to differentiate healthy individuals from EOC patients. (D) Binomial logistic regression-based classifier indicates the diagnostic power of the panel of miR-7, miR-429, miR-25 and miR-93, with a sensitivity of $93 \%$ and a specificity of $92 \%$ to distinguish healthy women from EOC patients. (E) The table summarises sensitivities and specificities of miR-7, miR-25, miR-93 and miR-429 and their combination.

We also compared the relative concentrations of circulating miR-7, miR-16, miR-25, miR-93, miR-182, miR-376a and miR-429 in the serum of EOC patients with their clinically relevant patient characteristics (grading, lymph node status and FIGO stages). Supplementary Table $\mathrm{S} 1$ summarises the $P$ values of the miRNA variables between different patient subgroups and control group. Our findings revealed the significant difference of the levels of miR-7 between healthy women and EOC patients $(P=0.001$, Figure 1A). However, a closer examination showed that the levels of circulating miR-7 were mainly increased in patients with lymph node metastases $(P=0.0001$, Figure $2 \mathrm{~B})$ and FIGO stage III-IV $(P=0.0001$, Figure $2 \mathrm{~A})$ and differed between FIGO I-II and FIGO III-IV $(P=0.011)$. On the other hand, the levels of miR-7 were increased in both subgroups of patients with lower (low grade; $P=0.0003$ ) or higher (high grade; $P=0.001$ ) grading compared with healthy women (supplementary Table S1). The serum levels of miR-376a were only associated with FIGO III-IV $(P=0.02)$ but not with FIGO I-II. In addition, the slightly higher serum levels of miR-376a observed in EOC patients compared with healthy women $(P=0.049$, Figure $1 \mathrm{~A})$ may be due to the higher levels of miR-376a in patients with FIGO III-IV $(P=0.02$, Figure $2 \mathrm{~A}$ ). These findings indicate that changes in the levels of miR-7 and miR-376a particularly occurred in advanced cancer (with distant metastases).

As shown in Figure 2A, the serum levels of miR-429 significantly differed between patients with FIGO I-II and those with FIGO III-IV $(P=0.001)$. There was a continuous rise of serum levels of miR-429 from healthy women over patients with FIGO I-II $(P=0.002)$ to patients with FIGO III-IV $(P=0.0001)$. ROC analyses showed that AUC values of CA125 and miR-429 were 0.822 and 0.704 (Figure 2D), respectively, showing the significant difference of their levels between EOC patients with FIGO I-II and FIGO III-IV. Using binary regression, the combination of miR-429 and CA125 values negligibly increased the AUC value of CA125 (from 0.822 to 0.826; Figure 2D). Although this combination slightly improved the sensitivity of CA125 (from 0.776 to 0.806 ), no change in their specificity could be observed (0.778) to distinguish FIGO I-II from FIGO III-IV (Figure 2E).

No correlations of miR-25 and miR-93 could be detected with the clinicopathological data. Both miRNAs are located in the same chromosomal cluster and display a similar deregulated expression pattern in the serum of EOC patients. In all subgroups, their levels were decreased compared with healthy women but could not differ between the analogous subgroups (Supplementary Table S1).

Finally, significant associations of serum levels of circulating miR-16 $(P=0.042), \quad m i R-376 a \quad(P=0.023)$ and miR-429 $(P=0.005)$ could be detected for gross residual tumour $(>1 \mathrm{~cm})$ but not for residual tumour of $<1 \mathrm{~cm}$ (Supplementary Table S1). Although the miRNAs were quantified in preoperative serum samples, this correlation of miRNA levels with the larger residual tumour after surgery could point to a higher tumour load. Moreover, the residual tumour correlated with tumour relapse $(P=0.0001)$ and a poor survival $(P=0.0001$, data not shown $)$.

Prognostic value of increased expression levels of serum miR429. Kaplan-Meier and log-rank models were carried out to assess the prognostic potential of our miRNA panel in EOC patients. The censored samples with a follow-up of $<6$ months were excluded for Kaplan-Meier and log-rank analyses. The median follow-up time was 21 months (range: 1-176 months), while the median overall and disease-free survival times were 22 and 15 months, respectively. Median values of miRNAs were used for grouping the serum samples according to low and high expression levels. As shown in Figure 3, the serum concentrations of circulating miR-429 significantly correlated with overall survival $(P=0.011$, 

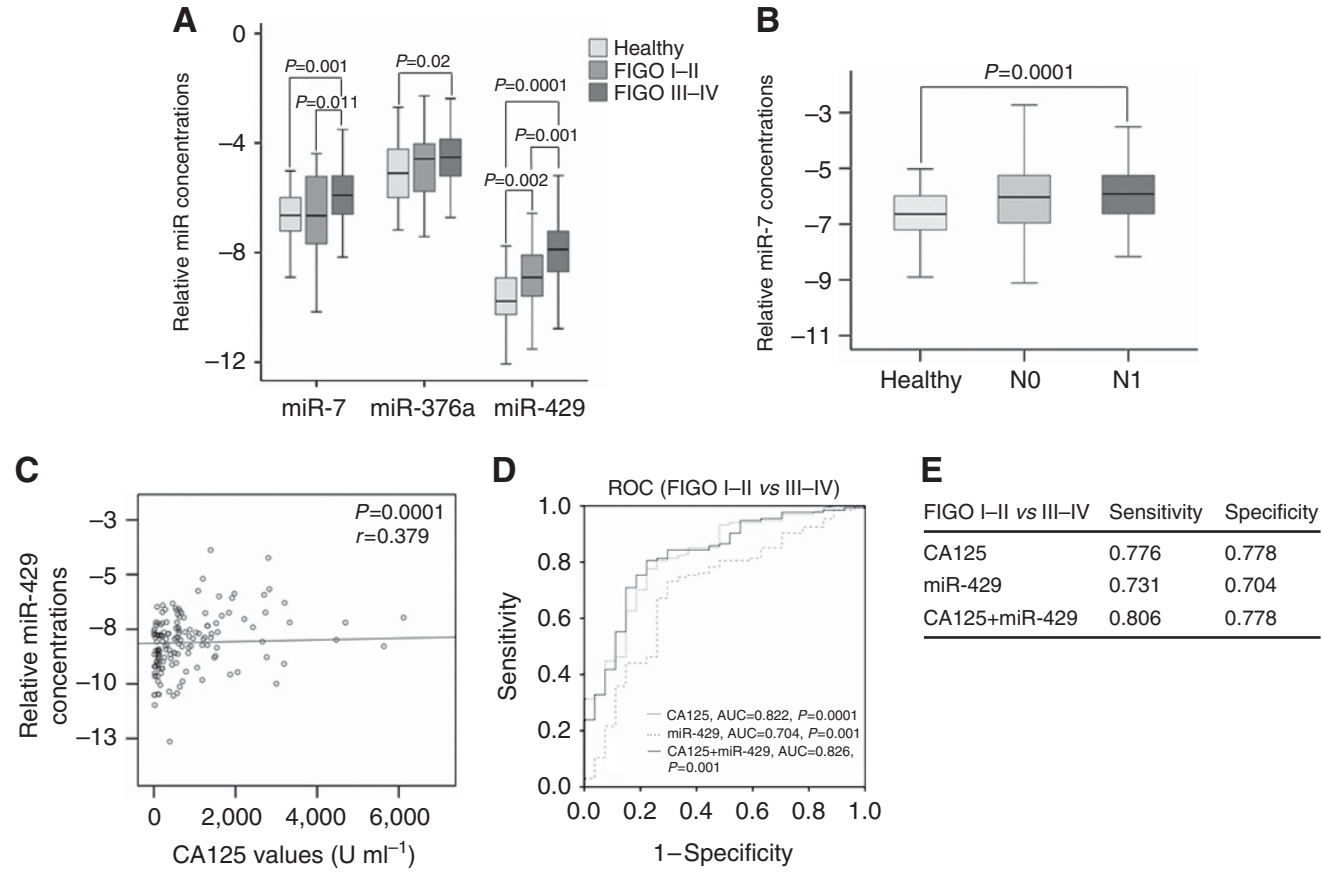

Figure 2. Correlations of serum levels of miR-7, miR-376a and miR-429 with patient parameters. (A) The box plot compares the concentrations of miR-7, miR-376a and miR-429 in the serum of healthy women $(n=66)$ with EOC patients with FIGO I-II $(n=32)$ and FIGO III-IV ( $n=147)$. (B) The box plots compare the miR-7 concentrations in the serum of healthy women $(n=66)$ with EOC patients with lymph-node negative (NO, $n=51)$ and lymph-node positive (N1, $n=88$ ) patients. (C) The scatter plot shows the relative miR-429 concentrations vs the CA125 values in EOC patients. (D) The ROC analyses show the sensitivities and specificities of CA125, miR-429 and their combination to differentiate FIGO I-II from FIGO III-IV. (E) The table summarises sensitivities and specificities of CA125, miR-429 and their combination.

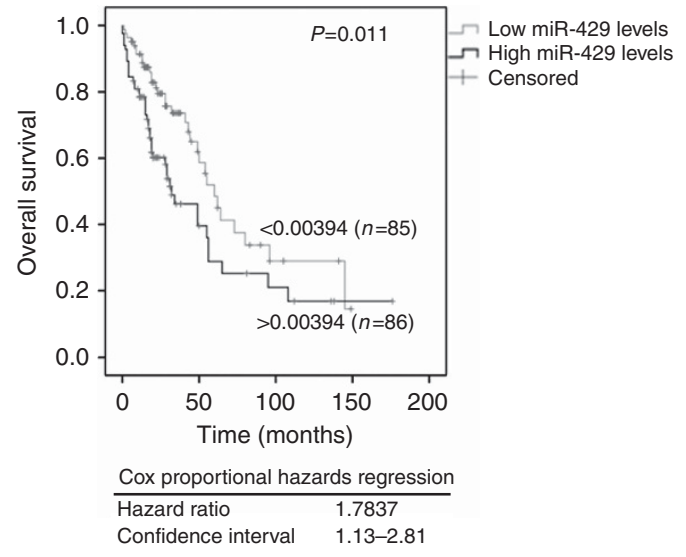

Figure 3. Serum levels of miR-429 significantly correlate with overall survival. Univariate Kaplan-Meier survival curves related to low and high concentrations of circulating miR-429 in the serum of EOC patients. The median value of 0.00394 was used for grouping the serum samples according to low $(n=85)$ and high $(n=86)$ transcript levels.

log-rank test). Univariate analysis with the Cox proportional hazards showed that serum miR-429 was a predictive factor (HR: 1.7837, 95\% CI: 1.13-2.81). Performing multivariate Cox analyses, the status of miR-429 expression along with FIGO stage $(P=0.031$, HR: 95\% CI, 0.383-0.964,), tumour residual ( $P=0.015$, HR: 95\% CI, 0.361-0.894) and FIGO stage plus tumour residual $(P=0.027$, HR: 95\% CI, 0.380-0.929) was an independent predictor of overall survival in EOC patients. The other circulating miRNAs showed no prognostic significance in our analyses.

Evaluation of miRNA data in the subgroup of high-grade, serious EOC patients. To validate the above results, we further examined the miRNA levels in 120 restricted EOC patients with high-grade cancer, the most common histological subtype, and found that the results were similar to those of the whole patient cohort. The detailed characteristics of high-grade EOC patients are summarised in Supplementary Table S3. In line with the findings derived from the whole EOC patient cohort $(n=180)$, the levels of miR-7 $(P=0.0001)$, miR-25 $(P=0.0001)$, miR-93 $(P=0.0001)$ and miR-429 $(P=0.0001)$ were deregulated in high-grade EOC patients compared with those of healthy women (Figure 4A), whereas the levels of miR-16, miR-182 and miR-376a showed no difference between both cohorts (Supplementary Table S2). In this high group, miR-7, miR-376a and miR-429 shared similar expression patterns with FIGO stages. As shown in Figure $4 \mathrm{~B}$, increased levels of miR-7 $(P=0.0001)$, miR-376a $(P=0.046)$ and miR-429 $(P=0.0001)$ correlated with FIGO III-IV compared with healthy women and showed a significant difference between FIGO I-II and FIGO III-IV $(P=0.006, P=0.026, P=0.0001$, respectively). The increased levels of miR-429 in high-grade EOC patients were still significantly associated with increased CA125 values $(P=0.001$, $r=0.305$, Figure 4C), similar to those observed in the whole patient cohort. In addition, the levels of miR-7 and miR-429 were upregulated in both subgroups of negative and positive lymph node statuses $(P=0.027, \quad P=0.002 ; \quad P=0.0001, \quad P=0.0001$; respectively). In contrast, the levels of miR-25 and miR-93 were decreased in the subgroups of FIGO I-II and FIGO III-IV ( $P=0.0001, P=0.0001 ; P=0.0001, P=0.0001$; respectively) and both lymph node statuses $(P=0.0001, P=0.0001 ; P=0.0001$, $P=0.0001$; respectively; Supplementary Table S2). The levels of miR-429 levels showed a borderline significance with gross residual tumour ( $>1 \mathrm{~cm}, P=0.049$; Supplementary Table S2).

Whereas these results obtained from the high-grade group corresponded to the whole EOC patient cohort, however, the prognostic value of miR-429 detected in the whole EOC patient cohort (overall survival, $P=0.011$ ) could not be verified in higgrade serious ovarian cancer patients $(P=0.131$, data not shown). 


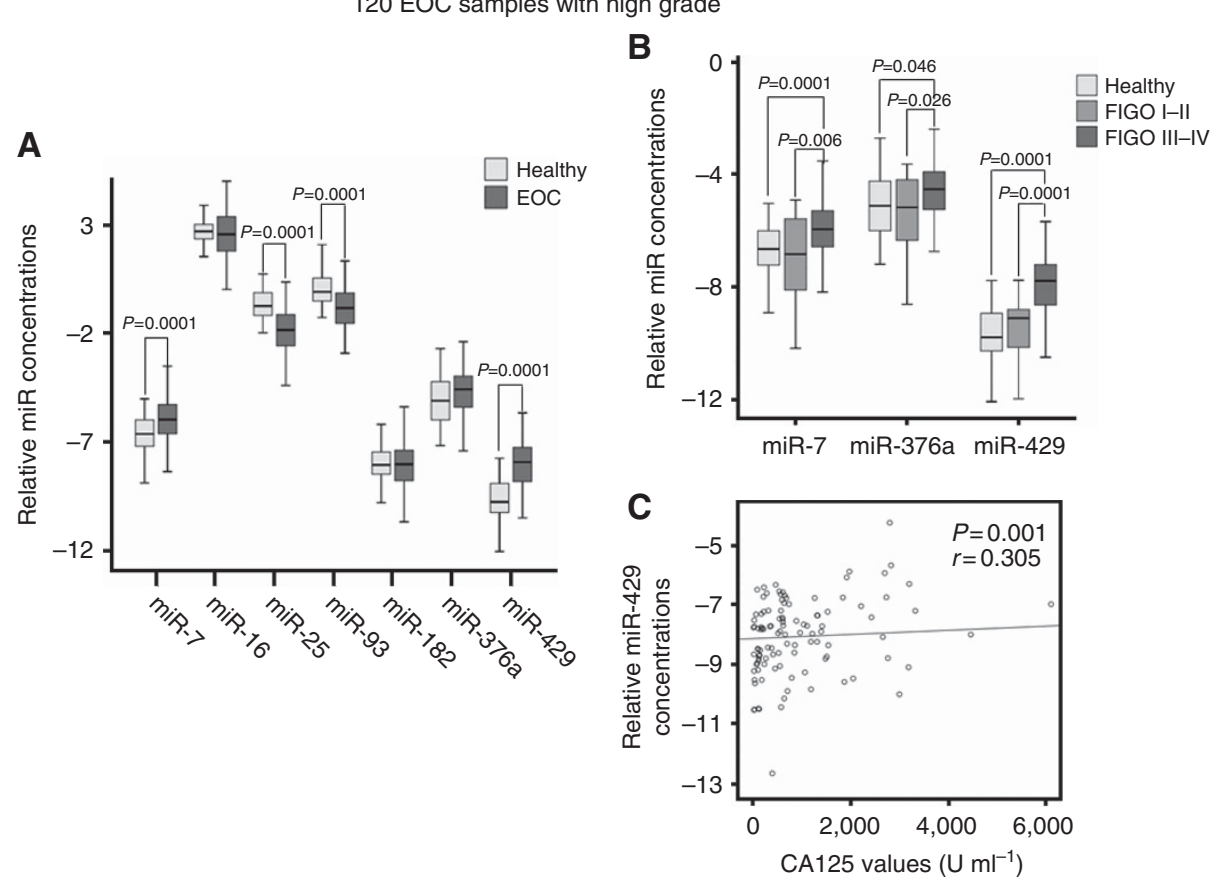

Figure 4. Evaluation of miRNA data in the subgroup of high grade, serious EOC patients. (A) The box plot compares the miRNA concentrations in the serum of healthy women $(n=66)$ with high grade EOC patients $(n=120)$. (B) The box plot compares the concentrations of miR-7, miR-376a and miR-429 in the serum of healthy women $(n=66)$ with high grade EOC patients with FIGO I-II $(n=17)$ and FIGO III-IV $(n=103)$. (C) The scatter plot shows the relative miR-429 concentrations vs the CA125 values in the patient cohort.

MiR-429 and miR-7 have no impact on cell proliferation and apoptosis of SKOV3 and OVCAR8 cells. For functional analyses, we selected miR-429 and miR-7 owing to their significant deregulation in EOC, in particular in advanced EOC. To determine their effect on cell proliferation and apoptosis, these analyses were carried out in SKOV3 and OVCAR8 cell lines. Cell proliferation was evaluated by an MTT assay. Both miRNAs did not affect cell proliferation (Supplementary Figure S2). For apoptosis, the transfected cells were additionally treated with the topoisomerase I inhibitor camptothecin, which is used in cancer chemotherapy and induces apoptosis. FACS analyses showed a camptothecinmediated apoptosis of OVCAR8 cells. Albeit higher doses of camptothecin were applied, the more aggressive SKOV3 cells did not display apoptotic features. Both miRNAs, miR-429 (Supplementary Figure S3A) and miR-7 (Supplementary Figure S3B) did not influence cell apoptosis.

MiR-429 and miR-7 affect the migration and invasion of SKOV3 and OVCAR8 cells. Our current findings show the association of miR-429 and miR-7 with lymph node and overt metastases of EOC patients, respectively. To examine whether this observation can be explained by an effect of both miRNAs on cell migration and invasion, respective assays were carried out in SKOV3 and OVCAR8 cells. Using transwell assays and performing transfection with miRNA mimics and inhibitors, we observed a decrease in cell migration (Figure $5 \mathrm{~A}, P=0.037$ ) and invasion (Figure $5 \mathrm{~B}, P=0.011$ ) after transfection of miR-429 mimic into more aggressive SKOV3 cells as compared with the cell line transfected with a negative control. No effect by the miR-429 inhibitor could be observed (Figures $5 \mathrm{~A}$ and $\mathrm{B}$ ). Inverse results could be observed for miR-7. In SKOV3 cells, miR-7 mimic could increase cell migration (Figure $5 \mathrm{C}, P=0.001$ ) and invasion (Figure 5D, $P=0.011$ ), whereas miR-7 inhibitor could only decrease migration (Figure 5C, $P=0.031$ ). In OVCAR8 cells, the values of migration were low (Figures $5 \mathrm{~A}$ and $\mathrm{C}$ ) and those of invasion were undetectable (data not shown). Only miR-429
(Figure 5A, $P=0.027$ ) and miR-7 (Figure 5C, $P=0.019$ ) inhibitors could increase and decrease OVCAR8 cell migration, respectively.

\section{DISCUSSION}

In the present study, we quantified the expression levels of miR-7, miR-16, miR-25, miR-93, miR-182, miR-376a and miR-429 in the preoperative serum of EOC patients, and because of their presence in advanced carcinomas, we carried out functional analyses using miR-429 and miR-7. Here we show that the serum levels of circulating miR-7, miR-25, miR-93 and miR-429 discriminated EOC patients from healthy women with a sensitivity of $93 \%$ and a specificity of $92 \%$. MiR-429 was an independent predictor of overall survival.

Our findings show for the first time that elevated serum amounts of miR-429, a member of the miR-200 family, may have diagnostic and prognostic relevance in EOC patients. Serum levels of this circulating miRNA were higher in EOC patients than in healthy women and rise progressively from healthy women over patients with FIGO I-II to patients with FIGO III-IV. As a consequence, its increased levels were also significantly associated with poor overall survival and were confirmed as an independent prognostic factor in multivariate analysis. Our results are in line with a previous small pilot study on tumour tissue by Nam et al (2008a) who observed that, in 20 patients diagnosed with serous ovarian carcinoma, higher expression levels of miR-429 were significantly associated with a poor prognosis. However, this miRNA was reported to also have a tumour-suppressor function (Hu et al, 2009a; Zhu et al, 2014). Hu et al (2009a) detected a significant association of low-level expression of miR-429 in tumour tissue, with poor overall survival of 55 advanced EOC patients. Supporting our hypothesis that miR-429 has also an oncogenic role, two recent studies have reported that miR-429 can promote the carcinogenesis of hepatocellular carcinoma (Huang et al, 2013) and colorectal cancer (Li et al, 2013). In these both 
A
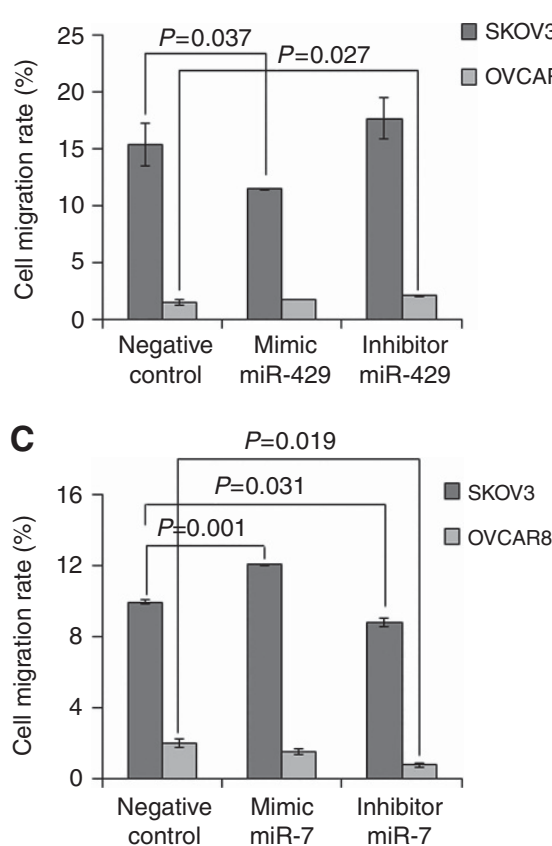

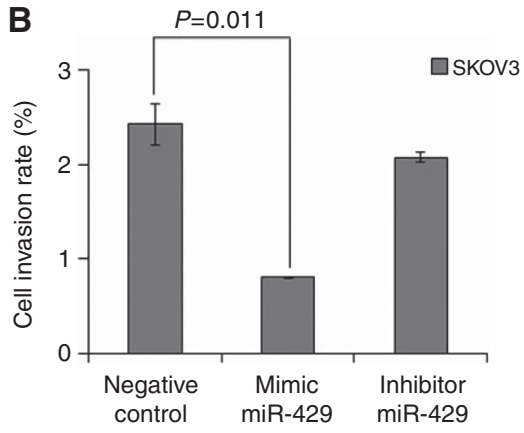

D

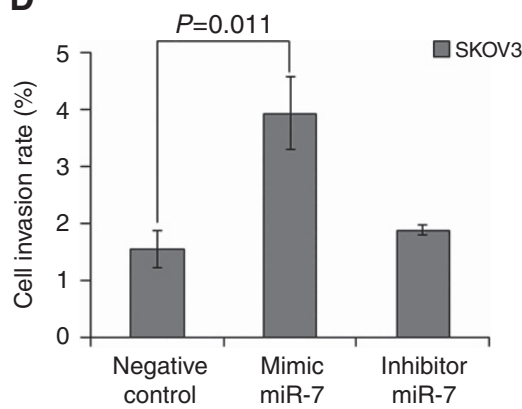

Figure 5. MiR-429 and miR-7 affect cell migration and invasion of SKOV3 and OVCAR8 cells. Cells were transfected with a negative (scrambled) control, (A, B) miR-429 or (C, D) miR-7 mimics and inhibitors, added to the upper transwell chambers and allowed to migrate or invade.

cancer types, miR-429 expression was also upregulated, and its high expression in tissues was significantly associated with advanced cancers and poor prognosis (Huang et al, 2013; Li et al, 2013). Moreover, we demonstrate the correlation of serum miR-429 with CA125 values. Patient subgroups harbouring normal and threshold CA125 values had about two- and three-fold higher mean serum levels of miR-429 than healthy women, respectively. To sum up, our findings show the potential clinical relevance of miR-429 in EOC.

As a member of miR-200 family, it was reported that ectopic overexpression of miR-429 in mesenchymal-like EOC cells induced changes in functional characteristics consistent with MET, resulting in reversal of the mesenchymal phenotype (Gregory et al, 2008; Chen et al, 2011). Accumulating evidence indicates that miR-429 is also involved in regulating migration and invasion of different cancer types. Overexpression of miR-429 suppressed migration and invasion of breast, colorectal and esophageal cancer cell lines (Wang et al, 2013; Sun et al, 2014; Ye et al, 2015). In addition to these cancer types, we demonstrate that miR-429 is also able to downregulate migration and invasion of the ovarian cancer cell line SKOV3 but has no effect on cell proliferation and apoptosis. The apparent discrepancy of the upregulation of serum miR-429 observed in our cohort of EOC patients with FIGO I-II and FIGO III-IV with its repression of cell migration and invasion in SKOV3 cells can be explained that miR-429 can act as an oncogene and a tumour-suppressor gene. MiR-429 could be expressed in a wavelike manner during carcinogenesis, so that it is upregulated in EOC patients with primary disease and patients with distant metastases but is possibly downregulated during the process of migration and invasion.

Regarding miR-7, our findings show that its concentrations were mainly increased in the serum of EOC patients with FIGO III-IV stage and lymph node-positive patients compared with the analogous counterparts. We also show that miR-7 could stimulate migration and invasion of SKOV3 cells, indicating that upregulation of circulating miR-7 may particularly be a diagnostic factor for advanced EOC patients. MiR-7 may also be involved in progression of other cancer types. It was reported that increased levels of miR-7 were connected with the metastatic capability of oestrogen receptor-positive primary breast cancer patients with lymph node-negative disease (Foekens et al, 2008). In renal cell carcinoma, miR-7 was also upregulated and its downregulation inhibited cell migration, suppressed proliferation and induced apoptosis of cancer cells (Yu et al, 2013). These and our data demonstrate that miR-7 may act as an oncogene. In contrast, other studies described miR-7 as a tumour suppressor in several human cancers, including breast cancer, lung cancer, glioblastoma and colorectal cancer, by targeting several proto-oncogenes (Kalinowski et al, 2014; Suto et al, 2015). These findings show its dual behaviour in different tumour types.

In our panel of circulating miRNAs, miR-25 and miR-93 were downregulated. Both miRNAs are located in the same chromosomal cluster and probably therefore displayed a similar aberrant expression pattern. In all subgroups of EOC patients, their levels were decreased compared with healthy women. To date, the expression levels of miR-25 have only been quantified in EOC tissues. Contrary to the diagnostic potential of decreased serum levels of miR-25 detected in our present study, recent publications reported an oncogenic role of increased serum levels of miR-25 in EOC tissue (Zhang et al, 2012; Wang et al, 2014b). Moreover, it was reported that the levels of miR-93 were increased in the serum of EOC patients (Ji et al, 2014). However, the patient cohort was much smaller than ours, and the authors used miR-16 as a reference to normalise their data, which has been shown to be often deregulated in other tumours (Ge et al, 2014; Wang et al, 2014a; Navarro et $a l, 2015)$. In contrast to that study and in line to our findings, it was also shown that the expression levels of miR-93 were significantly lower in primary serous EOC tissues than normal tissues (Theriault et al, 2014).

Finally, we also examined the miRNA levels in patients with high-grade serous ovarian cancer, the most common histological subtype. In line with our results derived from the whole patient cohort, including low- and high-grade EOC, miR-7, miR-25, miR93 and miR-429 were still significantly deregulated in this subgroup. Moreover, the significant associations of miR-7, miR25, miR-93 and miR-429 with FIGO stages detected in the whole 
EOC patient cohort could also be confirmed in high-grade patients, indicating the diagnostic power of these miRNAs. However, the prognostic value of miR-429 detected in the whole EOC patient cohort could not be verified in high-grade serious ovarian cancer patients.

To sum up, these data show that both, oncogenic and tumour inhibitory, traits can be ascribed to a miRNA, as well as to the miRNAs analysed and discussed in our study. These contradictory behaviours of miRNAs as either an oncogene or tumour suppressor can be explained by the imperfect complementarity of the interactions between miRNAs and their target genes. MiRNAs bind to the $3^{\prime}$ UTRs of their target genes leading to mRNA degradation or repression of translation. However, the bindings are not always completely complementary. Therefore, a specific miRNA can regulate hundreds of genes and a specific gene can be regulated by numerous miRNAs at posttranscriptional levels (Kim, 2005). The different expression levels and functions of miR7 and miR-429 may be further dependent on the diversity of cellular context in different cancer types, contributing to the bilateral regulations by some miRNAs (Yu et al, 2013). These argumentations document that the underlying mechanisms by which miRNAs act as an oncogene or tumour suppressor to promote or suppress cancer development and progression, respectively, are still largely unknown. Disadvantage for such analyses would be, if they are caused by the investigations of different tumour materials and the use of different normalisation strategies.

We show that the serum levels of circulating miR-7, miR-25, miR-93 and miR-429 were significantly deregulated in the serum of EOC patients. The upregulated expression of miR-7 was associated with EOC progression, potentially owing to cell migration and invasion. In contrast to some other studies (Nam et al, 2008b; $\mathrm{Hu}$ et al, 2009b) showing discrepant findings on miR-429 either as tumour suppressor or oncogene, we found that this miRNA can act as an oncogene (in vivo) and a tumour-suppressor gene (in vitro), presumably owing to its wavelike features during primary disease, migration/invasion and metastasis. However, to confirm the clinical relevance of these miRNAs, long-term follow-up studies of large patient cohorts and further functional analyses of these miRNAs are planned.

\section{ACKNOWLEDGEMENTS}

This study was supported by China Scholarship Council (201206170034), Else Kröner-Fresenius-Stiftung, Bad Homburg (2014-A106) and European Research Council Advanced Investigator Grant (ERC-2010 AsG 20100317 DISSECT).

\section{CONFLICT OF INTEREST}

The authors declare no conflict of interest.

\section{REFERENCES}

Bartel DP (2009) MicroRNAs: target recognition and regulatory functions. Cell 136(2): 215-233.

Chen J, Wang L, Matyunina LV, Hill CG, McDonald JF (2011) Overexpression of miR-429 induces mesenchymal-to-epithelial transition (MET) in metastatic ovarian cancer cells. Gynecol Oncol 121(1): 200-205.

Cheng AM, Byrom MW, Shelton J, Ford LP (2005) Antisense inhibition of human miRNAs and indications for an involvement of miRNA in cell growth and apoptosis. Nucleic Acids Res 33(4): 1290-1297.

Cui J (2015) MiR-16 family as potential diagnostic biomarkers for cancer: a systematic review and meta-analysis. Int J Clin Exp Med 8(2): 1703-1714.
Davidson B, Tropé CG, Reich R (2014) The clinical and diagnostic role of microRNAs in ovarian carcinoma. Gynecol Oncol 133(3): 640-646.

Eichelser C, Stückrath I, Müller V, Milde-Langosch K, Wikman H, Pantel K, Schwarzenbach H (2014) Increased serum levels of circulating exosomal microRNA-373 in receptor-negative breast cancer patients. Oncotarget 5(20): 9650-9663.

Foekens JA, Sieuwerts AM, Smid M, Look MP, de Weerd V, Boersma AW, Klijn JG, Wiemer EA, Martens JW (2008) Four miRNAs associated with aggressiveness of lymph node-negative, estrogen receptor-positive human breast cancer. Proc Natl Acad Sci USA 105(35): 13021-13026.

Formosa A, Markert EK, Lena AM, Italiano D, Finazzi-Agro E, Levine AJ, Bernardini S, Garabadgiu AV, Melino G, Candi E (2014) MicroRNAs, miR-154, miR-299-5p, miR-376a, miR-376c, miR-377, miR-381, miR487 b, miR-485-3p, miR-495 and miR-654-3p, mapped to the $14 \mathrm{q} 32.31$ locus, regulate proliferation, apoptosis, migration and invasion in metastatic prostate cancer cells. Oncogene 33(44): 5173-5182.

Ge W, Yu DC, Li QG, Chen X, Zhang CY, Ding YT (2014) Expression of serum miR-16, let-7f, and miR-21 in patients with hepatocellular carcinoma and their clinical significances. Clin Lab 60(3): 427-434.

Gregory PA, Bert AG, Paterson EL, Barry SC, Tsykin A, Farshid G, Vadas MA, Khew-Goodall Y, Goodall GJ (2008) The miR-200 family and miR-205 regulate epithelial to mesenchymal transition by targeting ZEB1 and SIP1. Nat Cell Biol 10(5): 593-601.

Heneghan HM, Miller N, Lowery AJ, Sweeney KJ, Newell J, Kerin MJ (2010) Circulating microRNAs as novel minimally invasive biomarkers for breast cancer. Ann Surg 251(3): 499-505.

Hu X, Macdonald DM, Huettner PC, Feng Z, El Naqa IM, Schwarz JK, Mutch DG, Grigsby PW, Powell SN, Wang X (2009a) A miR-200 microRNA cluster as prognostic marker in advanced ovarian cancer. Gynecol Oncol 114(3): 457-464.

Hu X, Macdonald DM, Huettner PC, Feng Z, El Naqa IM, Schwarz JK, Mutch DG, Grigsby PW, Powell SN, Wang X (2009b) A miR-200 microRNA cluster as prognostic marker in advanced ovarian cancer. Gynecol Oncol 114(3): 457-464.

Hu Z, Dong J, Wang LE, Ma H, Liu J, Zhao Y, Tang J, Chen X, Dai J, Wei Q, Zhang C, Shen H (2012) Serum microRNA profiling and breast cancer risk: the use of miR-484/191 as endogenous controls. Carcinogenesis 33(4): $828-834$.

Huang XY, Yao JG, Huang HD, Wang C, Ma Y, Xia Q, Long XD (2013) MicroRNA-429 modulates hepatocellular carcinoma prognosis and tumorigenesis. Gastroenterol Res Pract 2013: 804128.

Jayson GC, Kohn EC, Kitchener HC, Ledermann JA (2014) Ovarian cancer. Lancet 384(9951): 1376-1388.

Ji T, Zheng ZG, Wang FM, Xu LJ, Li LF, Cheng QH, Guo JF, Ding XF (2014) Differential microRNA expression by Solexa sequencing in the sera of ovarian cancer patients. Asian Pac J Cancer Prev 15(4): 1739-1743.

Joosse SA, Muller V, Steinbach B, Pantel K, Schwarzenbach H (2014) Circulating cell-free cancer-testis MAGE-A RNA, BORIS RNA, let-7b and miR-202 in the blood of patients with breast cancer and benign breast diseases. Br J Cancer 111(5): 909-917.

Kalinowski FC, Brown RAM, Ganda C, Giles KM, Epis MR, Horsham J, Leedman PJ (2014) microRNA-7: a tumor suppressor miRNA with therapeutic potential. Int J Biochem Cell Biol 54(0): 312-317.

Kim VN (2005) MicroRNA biogenesis: coordinated cropping and dicing. Nat Rev Mol Cell Biol 6(5): 376-385.

Komatsu S, Ichikawa D, Hirajima S, Kawaguchi T, Miyamae M, Okajima W, Ohashi T, Arita T, Konishi H, Shiozaki A, Fujiwara H, Okamoto K, Yagi N, Otsuji E (2014) Plasma microRNA profiles: identification of miR-25 as a novel diagnostic and monitoring biomarker in oesophageal squamous cell carcinoma. Br J Cancer 111(8): 1614-1624.

Li BS, Zuo QF, Zhao YL, Xiao B, Zhuang Y, Mao XH, Wu C, Yang SM, Zeng H, Zou QM, Guo G (2015) MicroRNA-25 promotes gastric cancer migration, invasion and proliferation by directly targeting transducer of ERBB2, 1 and correlates with poor survival. Oncogene 34(20): 2556-2565.

Li J, Du L, Yang Y, Wang C, Liu H, Wang L, Zhang X, Li W, Zheng G, Dong Z (2013) MiR-429 is an independent prognostic factor in colorectal cancer and exerts its anti-apoptotic function by targeting SOX2. Cancer Lett 329(1): 84-90.

McDermott AM, Kerin MJ, Miller N (2013) Identification and validation of miRNAs as endogenous controls for RQ-PCR in blood specimens for breast cancer studies. PLoS One 8(12): e83718.

Meinhold-Heerlein I, Hauptmann S (2014) The heterogeneity of ovarian cancer. Arch Gynecol Obstet 289(2): 237-239. 
Nam EJ, Yoon H, Kim SW, Kim H, Kim YT, Kim JH, Kim JW, Kim S (2008a) MicroRNA expression profiles in serous ovarian carcinoma. Clin Cancer Res 14(9): 2690-2695.

Nam EJ, Yoon H, Kim SW, Kim H, Kim YT, Kim JH, Kim JW, Kim S (2008b) MicroRNA expression profiles in serous ovarian carcinoma. Clin Cancer Res 14(9): 2690-2695.

Navarro A, Diaz T, Tovar N, Pedrosa F, Tejero R, Cibeira MT, Magnano L, Rosinol L, Monzo M, Blade J, Fernandez de Larrea C (2015) A serum microRNA signature associated with complete remission and progression after autologous stem-cell transplantation in patients with multiple myeloma. Oncotarget 6(3): 1874-1883.

Schwarzenbach H, Hoon DS, Pantel K (2011) Cell-free nucleic acids as biomarkers in cancer patients. Nat Rev Cancer 11(6): 426-437.

Simpson RJ, Lim JW, Moritz RL, Mathivanan S (2009) Exosomes: proteomic insights and diagnostic potential. Expert Rev Proteomics 6(3): 267-283.

Smith AL, Iwanaga R, Drasin DJ, Micalizzi DS, Vartuli RL, Tan AC, Ford HL (2012) The miR-106b-25 cluster targets Smad7, activates TGF-[beta] signaling, and induces EMT and tumor initiating cell characteristics downstream of Six1 in human breast cancer. Oncogene 31(50): 5162-5171.

Stuckrath I, Rack B, Janni W, Jager B, Pantel K, Schwarzenbach H (2015) Aberrant plasma levels of circulating miR-16, miR-107, miR-130a and miR-146a are associated with lymph node metastasis and receptor status of breast cancer patients. Oncotarget 6(15): 13387-13401.

Sun Y, Shen S, Liu X, Tang H, Wang Z, Yu Z, Li X, Wu M (2014) MiR-429 inhibits cells growth and invasion and regulates EMT-related marker genes by targeting Onecut2 in colorectal carcinoma. Mol Cell Biochem 390(1-2): 19-30.

Suto T, Yokobori T, Yajima R, Morita H, Fujii T, Yamaguchi S, Altan B, Tsutsumi S, Asao T, Kuwano H (2015) MicroRNA-7 expression in colorectal cancer is associated with poor prognosis and regulates cetuximab sensitivity via EGFR regulation. Carcinogenesis 36(3): 338-345.

Theriault BL, Basavarajappa HD, Lim H, Pajovic S, Gallie BL, Corson TW (2014) Transcriptional and epigenetic regulation of KIF14 overexpression in ovarian cancer. PLoS One 9(3): e91540.

Wagner U, Harter P, Hilpert F, Mahner S, Reuss A, du Bois A, Petru E, Meier W, Ortner P, Konig K, Lindel K, Grab D, Piso P, Ortmann O, Runnebaum I, Pfisterer J, Luftner D, Frickhofen N, Grunwald F, Maier BO, Diebold J, Hauptmann S, Kommoss F, Emons G, Radeleff B, Gebhardt M, Arnold N, Calaminus G, Weisse I, Weis J, Sehouli J, Fink D, Burges A, Hasenburg A, Eggert C (2013) S3-guideline on Diagnostics, Therapy and Follow-up of
Malignant Ovarian Tumours short version 1.0-AWMF registration number: 032/035OL. June 2013. Geburtshilfe Frauenheilkd 73(9): 874-889.

Wang H, Wang L, Wu Z, Sun R, Jin H, Ma J, Liu L, Ling R, Yi J, Wang L, Bian J, Chen J, Li N, Yuan S, Yun J (2014a) Three dysregulated microRNAs in serum as novel biomarkers for gastric cancer screening. Med Oncol 31(12): $1-7$.

Wang X, Meng X, Li H, Liu W, Shen S, Gao Z (2014b) MicroRNA-25 expression level is an independent prognostic factor in epithelial ovarian cancer. Clin Transl Oncol 16(11): 954-958.

Wang Y, Li M, Zang W, Ma Y, Wang N, Li P, Wang T, Zhao G (2013) MiR429 up-regulation induces apoptosis and suppresses invasion by targeting Bcl-2 and SP-1 in esophageal carcinoma. Cell Oncol (Dordr) 36(5): 385-394.

Xu X, Ayub B, Liu Z, Serna VA, Qiang W, Liu Y, Hernando E, Zabludoff S, Kurita T, Kong B, Wei J-J (2014) Anti-miR182 reduces ovarian cancer burden, invasion, and metastasis: an in vivo study in orthotopic xenografts of nude mice. Mol Cancer Ther 13(7): 1729-1739.

Ye ZB, Ma G, Zhao YH, Xiao Y, Zhan Y, Jing C, Gao K, Liu ZH, Yu SJ (2015) miR-429 inhibits migration and invasion of breast cancer cells in vitro. Int J Oncol 46(2): 531-538.

Yu Z, Ni L, Chen D, Zhang Q, Su Z, Wang Y, Yu W, Wu X, Ye J, Yang S, Lai Y, Li X (2013) Identification of miR-7 as an oncogene in renal cell carcinoma. J Mol Histol 44(6): 669-677.

Zhang H, Zuo Z, Lu X, Wang L, Wang H, Zhu Z (2012) MiR-25 regulates apoptosis by targeting Bim in human ovarian cancer. Oncol Rep 27(2): 594-598.

Zhou X, Hu Y, Dai L, Wang Y, Zhou J, Wang W, Di W, Qiu L (2014) MicroRNA-7 inhibits tumor metastasis and reverses epithelialmesenchymal transition through AKT/ERK1/2 inactivation by targeting EGFR in epithelial ovarian cancer. PLoS One 9(5): e96718.

Zhu W, He J, Chen D, Zhang B, Xu L, Ma H, Liu X, Zhang Y, Le H (2014) Expression of miR-29c, miR-93, and miR-429 as potential biomarkers for detection of early stage non-small lung cancer. PLoS One 9(2): e87780.

This work is published under the standard license to publish agreement. After 12 months the work will become freely available and the license terms will switch to a Creative Commons AttributionNonCommercial-Share Alike 4.0 Unported License.

Supplementary Information accompanies this paper on British Journal of Cancer website (http://www.nature.com/bjc) 